\title{
A Markov model for the effects of virological failure on HIV/AIDS progression in tuberculosis co-infected patients receiving antiretroviral therapy in a rural clinic in northern South Africa
}

\author{
C Shoko, ${ }^{1,2} \mathrm{PhD}$; D Chikobvu, ${ }^{1} \mathrm{PhD} ; \mathrm{P}$ O Bessong, ${ }^{3} \mathrm{PhD}$ \\ ${ }^{1}$ Department of Mathematical Statistics and Actuarial Sciences, Faculty of Natural and Agricultural Sciences, University of the Free State, \\ Bloemfontein, South Africa \\ ${ }^{2}$ Department of Mathematics and Computer Sciences, Faculty of Agriculture and Natural Sciences, Great Zimbabwe University, Zimbabwe \\ ${ }^{3}$ HIV/AIDS and Global Health Research Programme, Department of Microbiology, University of Venda, Thohoyandou, South Africa
}

Corresponding author: C Shoko (claris.shoko@gmail.com)

\begin{abstract}
Background. The goal of antiretroviral therapy (ART) is to suppress viral replication to undetectable levels. These low viral load (VL) levels may not be attained in some patients, a situation representing potential virological failure during the course of treatment.

Objectives. To present the results of a Markov model exploring how virological failure and active tuberculosis (TB) affect the progression of HIV in patients on ART.

Methods. A continuous-time non-homogeneous Markov model was used to model the progression of HIV/AIDS in patients on combination ART (cART). We define seven states in our model. The first five states are based on VL levels and the other two are absorbing states: death and withdrawal from the study. The effects of TB co-infection, baseline VL, lactic acidosis and treatment failure on transition intensities were assessed.

Results. The model shows that VL-based transition intensities do not follow a constant rate; rather, there are two different trends in HIV/ AIDS progression. The first trend is an increase in the prevalence of state 1 (undetectable VL levels) in the first 0.5 years of treatment. The second trend follows thereafter and shows a slow decrease. Within the first 0.5 years of therapeutic intervention, the undetectable VL state is therefore attainable from any VL state. However, when virological failure occurs, there is an increased risk of death. Developing active TB while on cART increases the risk of viral rebound from undetectable levels to VLs between 50 and 10000 copies $/ \mathrm{mL}$ by $\sim 1.03$-fold. From a VL between 10000 and 100000 copies/mL, developing TB while on cART increases the rate of viral rebound by 2.5-fold. However, if TB is detected and treated at enrolment, rates of viral rebound from undetectable levels are reduced.

Conclusions. The model confirms that virological failure, coupled with developing active TB while on cART, increases mortality rates irrespective of patient CD4+ count status. It also suggests that while TB at the time of cART initiation does not increase the risk of viral rebound, development of active TB after cART initiation does increase this risk. These findings highlight the importance of strengthening VL monitoring, which should be performed every 2 months, especially in patients with TB, and addressing unsuppressed VLs appropriately if they are detected.
\end{abstract}

S Afr Med J 2020;110(4):313-319. https://doi.org/10.7196/SAMJ.2020.v110i4.13934

The Joint United Nations Programme on HIV/AIDS (UNAIDS) reported that $\sim 37$ million people were living with HIV globally in 2017, with an estimated 17 million accessing antiretroviral therapy (ART). ${ }^{[1]}$ South Africa (SA) has one of the highest burdens of HIV in the world. In 2017, UNAIDS reported that there were an estimated 7.1 million people living with HIV in SA, 270000 new HIV infections and 110000 AIDS-related deaths. Of individuals living with HIV, only $\sim 45 \%$ had suppressed viral loads (VLs). ${ }^{[1]}$ The SA comprehensive treatment plan involves an approach to ensure that $90 \%$ of individuals infected with HIV are detected, $90 \%$ of those diagnosed are treated, and viral suppression is attained in $90 \%$ of all those under treatment (the 90-90-90 objective).

HIV infection is characterised by progressive depletion of the CD4+ T-cell population. This reduction increases the risk of latent tuberculosis (TB) reactivation 20 -fold, ${ }^{[2]}$ and the risk of developing active $\mathrm{TB}$ has been shown to increase significantly shortly after initial infection. ${ }^{[3]}$ Martinson et al. ${ }^{[4]}$ showed that $60-80 \%$ of people with
TB in southern Africa are HIV-positive, emphasising that TB is an important common opportunistic infection among HIV-infected individuals.

The use of combination ART (cART) in HIV/AIDS patients is characterised by an increase in $\mathrm{CD} 4+$ cell counts and a decrease in VL to undetectable levels. ${ }^{[5]}$ However, management of HIV infection in TB co-infected patients has been a challenge, in part owing to drug interactions between rifampicin and non-nucleoside reverse transcriptase inhibitors and protease inhibitors and concerns about adherence and virological failure. ${ }^{[6]}$ In a 2001 report, the World Health Organization (WHO) emphasised the need to further strengthen TB control between 2002 and 2020, as otherwise a billion people will be newly infected with Mycobacterium tuberculosis, more than 150 million will develop active TB disease, and 36 million will die of TB. ${ }^{[7]}$ Day et al. ${ }^{[8]}$ suggested that active TB accelerates HIV disease progression, but not many data are available to confirm or refute this hypothesis. Day et al.$^{[8]}$ argued that although ART is 
required for a major effect on survival in HIV-infected individuals, prevention of TB is important for reduction of HIV-related morbidity and mortality. Virological failure and TB control therefore need to be considered in tandem to minimise the consequences of partial or incomplete viral suppression.

Virological failure is due to patient factors such as non-adherence to treatment, or ART regimen factors such as peripheral neuropathy and lactic acidosis, drug resistance or drug-drug interactions. Constant monitoring of VL levels (states) helps in avoiding the unnecessary switch to second-line therapy that could be made when clinical (WHO stage) and immunological (CD4+ cell counts) criteria alone are used. Routine VL monitoring helps in the early detection of virological failure ${ }^{[9]}$ as patients are observed making random transitions from one VL state to another.

The random movement from one VL state to another is regarded as a stochastic process. HIV/AIDS progression is divided into various states of the disease based on VL measurement, including the endpoints death state and withdrawal from study (loss to followup). Stochastic processes allow random movements between VL states before an HIV/AIDS patient is finally absorbed into the death state. ${ }^{[10]}$ Patients are monitored only at visit times, which means that the exact time the transition occurred is not known. ${ }^{[11]}$ When transition times are not known and interval-censored observations are present, homogeneous and non-homogeneous Markov processes are an important field of research into stochastic processes. ${ }^{[12]}$ Nonhomogeneous models are particularly important when transition rates between disease states are not constant but allowed to change with time to mimic better, observed reality.

The Markov model is an appropriate stochastic approach when the present state of the patient summarises all previous information (known as the history or natural filtration of the process). Time-homogeneous Markov models have been widely used in the modelling of various disease progressions such as cancer, ${ }^{[13,14]}$ stroke ${ }^{[15]}$ and diabetic retinopathy. ${ }^{[16]}$ However, the assumption of time-homogeneity is unrealistic in the sense that over long periods the diseases evolve, resulting in changes in transition intensities. The use of time-homogeneous models then puts severe limitations on disease history behaviour. In particular, when dealing with HIV, it is more realistic to assume that science and medicine evolve, so the rate at which severity of the disease changes in patients is likely to alter as newer medications improve the quality of their lives. ${ }^{[15]}$ The science and medicine evolution justifies the need for continuous-time nonhomogeneous models in analysing disease progressions. The problem can be addressed by using piecewise constant transition rate Markov models that preserve the tractability of the constant rates. ${ }^{[17]}$

In the present study, a non-homogeneous Markov process, using a piecewise constant transition rate model approach, was used to model the progression of HIV/AIDS in patients with TB co-infection. The states of the Markov process are based on VL measurements followed by absorbing states, death or withdrawal from study. The VL states are defined as follows: (1) VL $<50$ copies/mL; (2) $50 \leq V L$ $<10000$; (3) $10000 \leq V L<100000$; (4) $100000 \leq V L<500000$; and (5) $V L \geq 500000$. State 1 is accessible from any VL state. The effects of virological failure and TB, among others, on HIV/AIDS progression are assessed. The inclusion of the effects of virological failure on HIV progression in patients with TB co-infection prompted the current research and analysis, and also the use of a continuous-time non-homogeneous Markov model in which the undetectable VL is accessed from any of the five VL states.

The SA description of virological failure is as follows: (i) two consecutive VLs $>1000$ copies/mL after previous suppression; (ii) one VL >1 000 copies/mL after previous suppression followed by a change in treatment; and (iii) one VL $>1000$ copies $/ \mathrm{mL}$ after 6 months on ART without suppression. This is in line with the Adult Guideline from the National Department of Health, which proposed a virological failure threshold of 1000 copies $/ \mathrm{mL} .^{[18]}$

Although VL level monitoring may not be routinely available in resource-limited settings, this monitoring helps in the detection of treatment failure and in avoiding the unnecessary switch to secondline treatment that could take place when clinical and immunological criteria alone are used. ${ }^{[19]}$ Treatment failure is defined clinically as a new or recurrent event indicating severe immunodeficiency or immunological failure (persistent CD $4+$ T-cell counts $<100$ cells $/ \mu \mathrm{L}$ ) after 6 months of treatment.

\section{Objectives}

To report on the development of a continuous-time non-homogeneous Markov model with states based on VL to assess the progression of HIV/AIDS in patients on cART.

\section{Methods}

\section{Ethical considerations}

The data collection procedures used in this study were approved by the Research Ethics Committee of the University of Venda, SA (ref. no. SMNS/13/ MBY/01/0625), in accordance with the 1964 Declaration of Helsinki and its subsequent amendments. Additionally, permission to access health facilities was obtained from the Limpopo Provincial Department of Health, SA, and the collaborating health facilities. Informed consent was obtained from study participants prior to their involvement, and data obtained were stripped of personal identifiers to ensure anonymity and confidentiality of the participants.

\section{Data description}

The study cohort comprised 399 HIV/AIDS patients undergoing treatment follow-up at a rural wellness clinic in Bela Bela, northern SA. The data were collected from 2004 to 2009 . TB tests were carried out at enrolment and also at every follow-up visit. At the time of data extraction, patients with TB were on TB treatment. At enrolment, the baseline VL, baseline CD4+ cell count, and data on the presence of active TB were retrieved. In this study, a TB case was the outcome of a diagnostic process comprising a combination of laboratory (microscopy and culture) and clinical investigations.

Of the patients, 338 had a VL $>10000$ copies/mL at baseline and 55 a VL of $\leq 10000$ copies/mL; 353 patients had a baseline CD4+ cell count $\leq 350$ cells $/ \mu \mathrm{L}$ and 46 a baseline count $>350$ cells $/ \mu \mathrm{L}$. Females comprised $69.1 \%(n=276)$ of the cohort. The variable age had a skew value of -0.44 at baseline, an indication that the majority of the patients were young adults. The variables age at baseline, VL at baseline and CD4+ at baseline are described further in Table 1.

Of the 399 patients, 292 (73.2\%) had TB co-infection; 261 of the HIV/TB co-infected patients $(65.4 \%$ of the total) already had active TB when they were enrolled at the clinic, but 89 patients (22.3\%) developed active TB while on treatment. After treatment, 58 (22.2\%) of the patients who had been cured of TB became actively infected once more. Of the patients who were HIV/TB co-infected at enrolment, most $(n=168)$ were given initial combination therapy of stavudine (d4T)-lamivudine (3TC)-efavirenz (EFV); 76 received initial combination therapy of $\mathrm{d} 4 \mathrm{~T}-3 \mathrm{TC}$-nevirapine (NVP), 6 received zidovudine (AZT)-3TC-lopinavir/ritonavir (LPV/r), 5 received abacavir (ABC)-AZT-3TC, and 4 received d4T-3TC$\mathrm{LPV} / \mathrm{r}$. These drugs belong to the nucleoside reverse transcriptase inhibitor (NRTI) class. 
Table 1. Descriptive summary of age, VLBL and CD4+BL

\begin{tabular}{llll}
\hline & Age (years) & VLBL (copies/mL) & CD4+BL $($ cells/ $\boldsymbol{\mu L})$ \\
\hline Minimum & 15 & $<50$ (undetectable) & 16 \\
First quartile & 32 & 21334 & 38 \\
Mean & 38.3 & 138208 & 163 \\
Median & 39 & 67995 & 116 \\
Third quartile & 47 & 201445 & 206 \\
Maximum & 77 & $>500000$ & 1202 \\
VLBL = viral load at baseline; $\mathrm{CD} 4+\mathrm{BL}=\mathrm{CD} 4+$ cell count at baseline. & &
\end{tabular}

Although NRTIs are relatively affordable, they cause varying degrees of myopathy and neuropathy in the long term. ${ }^{[20]}$ AZT causes myopathy, didanosine (ddI) and 3TC cause neuropathy, and d4T causes neuropathy, myopathy and lactic acidosis. Patients may also experience virological failure. In this article, treatment failure is defined clinically and immunologically. The effect of lactic acidosis and peripheral neuropathy, together with other covariates, on the progression of HIV is analysed.

\section{Limitations of the data}

This was a retrospective set of data. Information on other comorbidities or opportunistic infections in the patients was not available, and the observations should be understood in the context of these limitations. The intended outcome of cART is to suppress VLs to undetectable levels, in the absence or presence of comorbidities.

\section{Piecewise constant transition rate Markov model}

Modelling the non-homogeneous Markov model can easily be done using a piecewise constant intensities approach. According to SaintPierre et al. ${ }^{[21]}$ in an analysis of asthma, this approach involves the inclusion of time-dependent covariates in a Markov model, making it easier to deal with non-homogeneous Markov models. The approach partitions the time axis into $r$ continuous and disjoint intervals

$\left[\tau_{l-1}, \tau_{l}\right)$, where $l=1, \ldots, r+1$ and $\tau_{r+1}=\infty$, and assumes constant transition intensities in different time intervals.

Consider a vector $z^{*}(t)=\left(z_{0}^{*}(t), z_{1}^{*}(t), \ldots, z_{r}^{*}(t)\right)^{\prime}$ of artificially time-dependent covariates defined as:

$$
\begin{gathered}
z_{0}^{*}(t)=0, \forall t \\
z_{l}^{*}(t)= \begin{cases}0 ; & \text { if } \tau_{0} \leq t<\tau_{l}, \\
1 ; & \text { if } t \geq \tau_{l}\end{cases}
\end{gathered}
$$

where $l=1, \ldots, r+1$. The model with transition intensities is as follows:

$$
q_{i j}\left(t \mid z^{*}(t)\right)=q_{i j}^{(0)} \exp \left\{\left(\beta_{i j}^{*}\right)^{\prime} z^{*}(t)\right\}, i \neq j
$$

This approach to non-homogeneity in a Markov process is a stepwise method that assumes constant transition intensities in different time intervals. The parameters of this model are the baseline

transition intensities $q_{i j}^{(0)}$, which represent transition intensities in the interval $\left[\tau_{0}, \tau_{1}\right)$, and the vector of regression coefficient $\beta_{i j}^{*}$ associated with artificially time-dependent covariates. For this model, transition intensities are step-functions of time and defined for each interval as follows:

$$
q_{i j}\left(t \mid z_{l}^{*}(t)\right)=\left\{\begin{array}{lr}
q_{i j}^{(0)} & \text { if } \tau_{0} \leq t<\tau_{1} \\
q_{i j}^{(1)}=q_{i j}^{(0)} \exp \left\{\beta_{i j, 1}^{*}\right\} ; & \text { if } \tau_{1} \leq t<\tau_{2} \\
\vdots & \\
q_{i j}^{(l)}=q_{i j}^{(0)} \exp \left\{\beta_{i j, 1}^{*} z_{1}^{*}+\beta_{i j, 2}^{*} z_{2}^{*}+\cdots+\beta_{i j, l}^{*} z_{l}^{*}\right\} ; & \text { if } t \geq \tau_{l}
\end{array}\right.
$$

for $l=1,2, \ldots, r$.

Incorporating the effects of covariates, represented by the vector $z$, the $q_{i i}\left(t \mid z_{l}{ }^{*}(t), \boldsymbol{z}\right)=q_{i j}^{(0)} \exp \left\{\beta_{i j, l}^{*}{ }^{\prime} \mathbf{z}_{l}{ }^{*}(t)+\beta_{i j}{ }^{\prime} \boldsymbol{z}\right\}$ where $\beta_{i j}$ is the log-linear effect relating to the instantaneous rate of transition from state $i$ to state $j$ to the covariate $z$.

Computing $P\left(0, t_{i}\right)_{\text {for a }} t_{i \text { in segment }} \tau_{l \text { entails multiplying }}$ all the transition matrices across the various intervals, as shown below:

$$
P\left(0, t_{i}\right)=\left[\pi_{l=1}^{l-1} P^{(b)}\left(\pi_{b}\right)\right] P^{(l)}\left(t_{(l-1)}, t_{j}\right)
$$

where $P^{(b)}$ is the transition probability matrix obtained using $q_{i j b \text { for the }} b^{t h}$ segment denoted by $\tau_{b}$. If subjects are observed on an equal-spaced grid and segments are divided up along these

time points, then $p_{i j}\left(0, t_{i}\right)_{\text {would simply be the }}(i j)^{t h}$ element of the matrix in the above equation. When data are not equally spaced, then observations would be considered missing at the breakpoints. To resolve this, a model that accounts for all possible

pathways between the last observed state in the segment $b_{l-1}$ and the first observation in segment $b_{0}$ was suggested. For example, if a breakpoint $t^{\prime}$ is created between two points $t_{j}$ and $t_{k \text {, then via }}$ Chapman-Kolmogorov equations, the likelihood contribution from interval $\left(t_{j}, t_{k}\right)$ for individual $x$ can be found as:

$$
L_{x}=\sum_{l=1}^{k} P_{i l}^{(1)}\left(t_{j}, t^{\prime}\right) P_{l j}^{(2)}\left(t^{\prime}, t_{k}\right)
$$

for states $i$ and $j$.

\section{Model formulation}

Patients were put on cART at time $\mathrm{t}=0$. The patients were monitored after 3 months ( 0.25 years) of cART and thereafter at follow-up intervals of 6 months ( 0.5 years). Follow-up was done for a maximum 
of 5 years, but owing to some deaths and withdrawal cases associated with the data, the average follow-up time for each patient in the study was 3.5 years. At follow-up times, the effectiveness of cART was assessed by changes in the HIV VL. Attainment of a suppressed VL below the level of detection within the first 6 months indicated good adherence to treatment and effectiveness of cART. In this study, the level of detection was 50 viral RNA copies/mL.

VLs for each individual during the course of treatment were classified into states based on the severity of the patient's condition, as follows:

Viral load state $= \begin{cases}1, & \text { if } V L<50 \\ 2, & \text { if } 50 \leq V L<10000 \\ 3, & \text { if } 10000 \leq V L<100000 \\ 4, & \text { if } 100000 \leq V L<500000 \\ 5, & \text { if } V L \geq 500000 \\ 6, & \text { if dead } \\ 7, & \text { if loss to follow up }\end{cases}$

States $i=1,2,3,4,5$ are the live/transient states and states 6 and 7 are the absorbing states. Transitions from state $i$ to $i+c$, for $c>0$, represent disease progression to worse states and transitions from state $i$ to state $i-c$, for $c>0$, represent disease progression to better states. At $\mathrm{t}=0$ there were 2 patients in VL state $1 ; 42$ in state $2 ; 174$ in state 3; 135 in state 4 ; and 45 in state 5 . This confirms that at treatment initiation, most patients had a VL between 10000 and 100000 copies/mL (state 3).

Table 2 is a state table that shows the possible transitions, from state $i$ to state $j, j=i \pm c$, that occurred during the study period.

Table 2 shows that most states are accessible from each other. Of interest is the undetectable VL state (state 1), which is accessible from all transient states. Based on these transitions, the transition diagram in Fig. 1 is therefore proposed.

The arrows in the diagram represent possible transitions between states. Green arrows represent viral suppression. Blue arrows represent viral rebound and red arrows represent absorption into the death state. Orange arrows represent loss to follow-up. Transitions between states can be represented by the transition matrix below:

$$
Q(t)=\left(\begin{array}{ccccccc}
q_{11} & q_{12} & 0 & 0 & 0 & q_{16} & q_{17} \\
q_{21} & q_{22} & q_{23} & 0 & 0 & q_{26} & q_{27} \\
q_{31} & q_{32} & q_{33} & q_{34} & 0 & q_{36} & q_{37} \\
q_{41} & q_{42} & q_{43} & q_{44} & q_{45} & q_{46} & q_{47} \\
q_{51} & q_{52} & 0 & q_{54} & q_{55} & q_{56} & q_{57} \\
0 & 0 & 0 & 0 & 0 & 0 & 0 \\
0 & 0 & 0 & 0 & 0 & 0 & 0
\end{array}\right)
$$

The force of transition from state $i$ to $j$ is defined as:

$$
q_{i j}(t)=\left.\frac{d}{d t} p_{i j}(t)\right|_{t=0}=\lim _{\Delta t \rightarrow 0} \frac{p_{i j}(\Delta t)-\delta_{i j}}{\Delta t}
$$

$q_{i j}$, for $i=1, \ldots, 5$ and $j=1, \ldots, 7$, vary over time and satisfy the

following conditions: $\sum_{j \in X} q_{i j}(t)=0$ and

$q_{i i}(t)=-\sum_{i \neq j} q_{i j}(t)$. Once the transition intensities are estimated, the probabilities that state $j$ is next subject in the long run, on condition that the patient was in state $i$, is given as

$p_{i j}=\frac{q_{i j}}{\lambda_{i}}$, for each $i$ and $j$ where $\lambda_{i}=\sum_{j \in X} q_{i j}(t)$, such

\begin{tabular}{llllllll}
\multicolumn{7}{l}{ Table 2. State table, $\boldsymbol{n}$ transitions } \\
\cline { 2 - 8 } From $(\boldsymbol{i})$ & $\mathbf{1}$ & $\mathbf{2}$ & $\mathbf{3}$ & $\mathbf{4}$ & $\mathbf{5}$ & $\mathbf{6}$ & $\mathbf{7}$ \\
\hline $\mathbf{1}$ & 1185 & 94 & 22 & 2 & 0 & 22 & 53 \\
$\mathbf{2}$ & 198 & 105 & 27 & 4 & 2 & 14 & 24 \\
$\mathbf{3}$ & 105 & 71 & 34 & 2 & 0 & 27 & 14 \\
$\mathbf{4}$ & 55 & 70 & 8 & 2 & 4 & 17 & 0 \\
$\mathbf{5}$ & 13 & 22 & 0 & 8 & 0 & 3 & 2
\end{tabular}

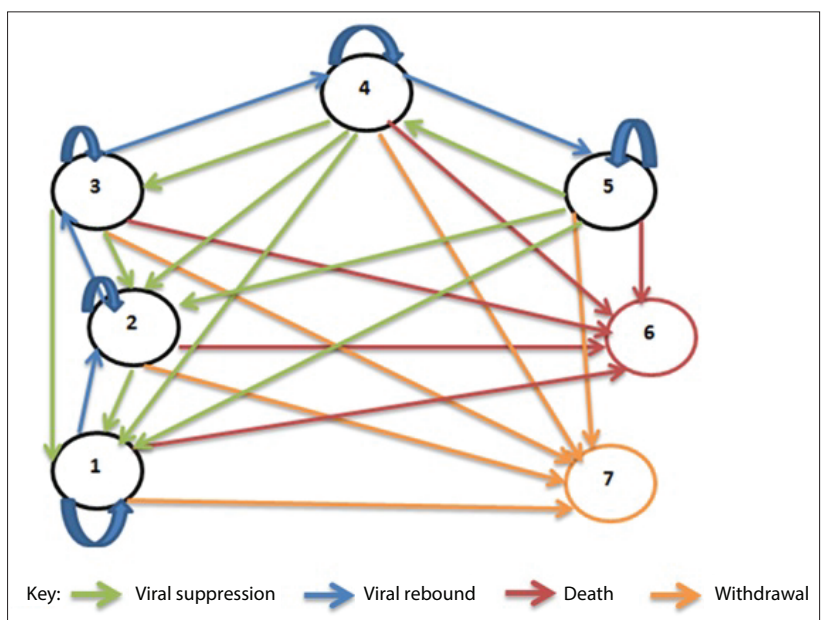

Fig. 1. Transition diagram.

that $i \neq j$ is the total time spent in state $i$ before a jump to state j. For example,

$$
p_{12}=\frac{q_{12}}{q_{12}+q_{16}+q_{17}}
$$

The effects of the covariates gender, age, virological failure (VF), treatment failure (TF), VL level baseline (VLBL), peripheral neuropathy (PN), having TB before enrolment (TBB4), developing TB while on ART (TBEN), lactic acidosis (LA) and the effect of time on transition intensities were assessed. Peripheral neuropathy and lactic acidosis are long-term effects of the NRTI class. Virological failure is defined as having a VL of at least 1000 copies $/ \mathrm{mL}$ in two consecutive visits or having a VL $>1000$ copies $/ \mathrm{mL}$ in the first 6 months of cART initiation. In this article, treatment failure is defined immunologically and clinically. These covariates are coded as follows:

$$
\begin{gathered}
\text { Age }=\left\{\begin{array}{l}
1, \leq 45 \text { years } \\
0,>45 \text { years }
\end{array}, \quad \mathrm{VF}=\left\{\begin{array}{l}
1, \text { Yes } \\
0, \text { No }
\end{array}, \quad \mathrm{TBB} 4=\left\{\begin{array}{l}
1, \text { Yes } \\
0, \text { No }
\end{array}, \quad \mathrm{LA}=\left\{\begin{array}{l}
1, \text { Yes } \\
0, \text { No }
\end{array}\right.\right.\right.\right. \\
\mathrm{TBEN}=\left\{\begin{array}{l}
1, \text { Yes } \\
0, \text { No }
\end{array}, \quad \text { Gender }=\left\{\begin{array}{l}
1, \text { male } \\
0, \text { female }
\end{array}, \quad \mathrm{TF}=\left\{\begin{array}{ll}
1, \text { Yes } \\
0, \text { No }
\end{array}, \quad \mathrm{PN}=\left\{\begin{array}{l}
1, \text { Yes } \\
0, \text { No }
\end{array}\right.\right.\right.\right.
\end{gathered}
$$

$\mathrm{VLBL}=\left\{\begin{array}{l}1,>10000 \text { copies } / \mu L \\ 0, \leq 10000 \text { copies } / \mu L\end{array}, \quad\right.$ Time $=\left\{\begin{array}{l}0 ; \text { if } 0 \leq t<0.5 \\ 1 ; \text { if } t \geq 0.5 \text { years }\end{array}\right.$

$\mathrm{CD} 4 \mathrm{BL}=\left\{\begin{array}{l}1, \leq 350 \text { cells } / \mathrm{mm}^{3} \\ 0,>350 \text { cells } / \mathrm{mm}^{3}\end{array}\right.$

So that the piecewise constant Markov model becomes:

$$
q_{i j}\left(t \mid \mathbf{z}_{l}^{*}(t), \mathbf{z}\right)=q_{i j}^{(0)} \exp \left\{\beta_{i j, l}^{*} \mathbf{z}_{l}{ }^{*}(t)+\beta_{i j}{ }^{\prime} \mathbf{z}\right\}
$$

where $\boldsymbol{z}=\left\{\begin{array}{c}\text { Age, Virological failure }(V L), T B \text { before treatment }(T B B 4), \\ \text { Lactic acidosis }(L A), T B \text { while on cART }(T B E N), \text { Gender, } \\ \text { Treatment failure }(T F), \text { Peripheral neuropathy }(P N), \\ \text { Viral load at baseline }(V L B L), \text { Time, CD }+ \text { at baseline }(C D 4 B L)\end{array}\right\}$ 


\section{Results}

In this section, a continuous-time non-homogeneous Markov model for the effects of time $t \geq 0.5$ years, VL baseline, gender, having TB co-infection, having active TB before enrolment, developing active TB on treatment, peripheral neuropathy, lactic acidosis, virological failure and treatment failure on virology is defined by the equation:

$$
\left.q_{i j}\left(t \mid z^{*}(t), \mathbf{z}\right)=q_{i j}^{(0)} \exp \left\{\boldsymbol{\beta}_{i j l}^{*}{ }^{\prime} \mathbf{z}_{l}^{*}(t)+\boldsymbol{\beta}_{i j}{ }^{\prime} \mathbf{z}\right]\right\}, i \neq j
$$

The parameter $q_{i j}^{(0)}$ is the baseline transition intensities for intervals

[0, 0.5 year], $\boldsymbol{\beta}_{i j l}^{*}$ is the vector of regression coefficients associated with the artificial time-dependent covariates

$$
\boldsymbol{z}_{l}^{*}=[1,0]^{\prime} \text { as defined earlier, and } \boldsymbol{\beta}_{i j} \text { is the vector of }
$$
regression coefficients associated with covariates $z=[\mathrm{VLBL}$, Gender, TB, TBEN, TBB4, PN, LA, VF, TF]'. The results from the fitted model are shown in Table 3.

Results show that when the VL of a patient is $<100000$ copies $/ \mathrm{mL}$, rates of viral suppression are higher than rates of viral rebound. The undetectable VL (state 1) is accessible from all the states. The rate of attainment of an undetectable VL depends on the condition of a patient. Patients with the highest viral copies/mL (state 5) have the lowest risk of attaining an undetectable VL, whereas patients with the lowest viral copies/mL (state 2) have the highest chance of doing so.

Developing active TB while on ART (TBEN) increases the rates of viral rebound from an undetectable level (state 1) to a VL measurement $>50$ copies $/ \mathrm{mL}$ and $<10000$ copies $/ \mathrm{mL}$ (state 2). If the TB is detected at enrolment (TBB4), there are reduced rates of viral rebound from state 1 to state 2 . However, detecting TB co-infection, be it at enrolment or during the course of treatment, reduces viral rebound from state 1 to state 2. Virological failure is experienced from state 1 to state 2 . Other factors that contribute to viral failure from undetectable levels are peripheral neuropathy and lactic acidosis. From 0.5 years of treatment and beyond, there is reduced viral rebound from undetectable levels. More time on cART therefore reduces rates of viral rebound.

Table 3 shows virological rebound from a VL state between 50 and 10000 copies/mL (state 2) to a VL state between 10000 copies/ $\mathrm{mL}$ and 100000 copies/mL (state 3). The virological failure is due to effects of developing TB on treatment (TBEN), having TB at enrolment (TBB4), lactic acidosis, treatment failure and having a VL baseline $>10000$ copies/mL at enrolment.

Patients mostly experience virological failure in state 4 (VL baseline between 100000 and 500000 copies $/ \mathrm{mL}$ ). These patients experience a viral rebound back to a VL level $>500000$ copies $/ \mathrm{mL}$ (state 5). Males are also at higher risk of experiencing a rebound from state 4 to state 5 compared with their female counterparts.

Results show an increased rate of mortality (state 6) from an undetectable VL (state 1) for patients who enrolled with a VL baseline $>10000$ copies $/ \mathrm{mL}$, patients who developed active TB while on cART, and patients who experienced virological failure. Patients who develop active TB while on cART have accelerated rates to mortality regardless of $\mathrm{CD} 4+$ status.

Next we estimate long-run probabilities of state $j$ being the next state given the condition that the patient was initially in state $i$, referred to as the jump process. This is when a Markov process is observed at the times it makes transitions to a new state. In other words, a jump process is a stochastic matrix $R$ of probabilities where each row sums to one on the state space $X_{t}$, which gives the conditional probability

\begin{tabular}{|c|c|c|c|c|c|c|c|c|c|c|c|}
\hline State $\boldsymbol{i}-\boldsymbol{j}$ & $\begin{array}{l}\text { Baseline } \\
\mathbf{Q}_{i j}{ }^{(0)}\end{array}$ & VLBL & Gender & TB & TBEN & TBB4 & PN & LA & VF & TF & $\begin{array}{l}\text { Time } \\
{[0.5, \infty]}\end{array}$ \\
\hline State $2-1$ & 2.333 & -0.063 & 0.191 & -1.624 & 0.216 & 0.973 & 0.767 & -0.038 & -1.337 & -0.353 & -1.003 \\
\hline State 3 - 1 & 0.966 & 0.424 & 0.479 & 0.419 & -0.504 & -0.121 & 1.523 & 1.346 & -1.091 & -0.585 & -2.719 \\
\hline State 4 - 1 & 0.545 & 0.145 & 0.259 & 0.137 & 0.413 & 0.576 & -0.014 & 0.517 & -0.124 & -0.119 & -0.778 \\
\hline State $5-1$ & 0.427 & 0.222 & -0.203 & -0.201 & 0.532 & -0.008 & -0.229 & 0.244 & -0.055 & -0.039 & -0.879 \\
\hline State $1-2$ & 0.410 & 0.017 & -0.016 & -0.539 & 0.032 & -0.139 & 0.116 & 0.18 & 1.985 & -0.08 & -1.394 \\
\hline State 3 - 2 & 2.775 & -0.022 & -0.660 & 0.460 & -0.625 & -0.832 & -0.601 & -0.298 & -0.232 & -1.046 & -2.041 \\
\hline State $4-2$ & 4.978 & 0.159 & -0.583 & -0.375 & -0.355 & 1.219 & -0.503 & 0.069 & -0.049 & 0.229 & -1.626 \\
\hline State 5 - 2 & 2.428 & 0.129 & -0.338 & 0.621 & 0.441 & 1.080 & 0.200 & -0.077 & 0.289 & -0.076 & -1.432 \\
\hline State 2 - 3 & 0.852 & 1.403 & -0.789 & -1.530 & 0.493 & 1.010 & -0.132 & 0.685 & 1.265 & 0.879 & -0.660 \\
\hline State 4 - 3 & 0.148 & 0.082 & -0.068 & -0.131 & -0.026 & -0.071 & 0.147 & -0.057 & -0.010 & -0.006 & -0.132 \\
\hline State $3-4$ & 0.439 & 0.315 & -0.195 & 0.516 & 0.896 & 0.590 & 0.452 & -0.803 & 0.339 & -0.522 & -0.023 \\
\hline State $5-4$ & 1.108 & 0.315 & 0.272 & -0.521 & 1.004 & -1.475 & -0.223 & -0.759 & -0.069 & -0.064 & -1.578 \\
\hline State 4 - 5 & 0.456 & 0.253 & 0.695 & -0.953 & -0.429 & -0.775 & -0.315 & -0.852 & 0.748 & -0.044 & -1.222 \\
\hline State $1-6$ & 0.010 & 0.809 & -0.742 & -1.451 & 0.560 & -2.716 & -1.120 & -1.316 & 0.024 & -0.103 & -2.345 \\
\hline State $2-6$ & 0.032 & 0.453 & 0.572 & -0.316 & 1.151 & -2.083 & -0.232 & -1.001 & -0.333 & -0.153 & -0.396 \\
\hline State $3-6$ & 0.040 & 0.064 & 0.116 & -0.163 & 0.280 & -0.915 & 0.006 & -0.404 & -0.290 & -0.201 & -0.434 \\
\hline State $4-6$ & 0.138 & 0.092 & 0.014 & -0.101 & 0.459 & -0.423 & -0.188 & -0.133 & -0.034 & -0.029 & 0.398 \\
\hline State 5 - 6 & 0.106 & 0.018 & 0.185 & -0.078 & 0.041 & -0.157 & -0.032 & -0.050 & -0.032 & -0.002 & -0.053 \\
\hline State $1-7$ & 0.037 & 0.564 & -0.577 & -0.990 & -0.081 & -1.572 & -1.518 & -1.733 & 0.087 & 0.235 & 0.776 \\
\hline State $2-7$ & 0.103 & -0.524 & -0.036 & 0.010 & 0.161 & 0.303 & -0.448 & -1.556 & 0.007 & -0.126 & -0.139 \\
\hline State 3 - 7 & 0.080 & -0.018 & 0.016 & -0.176 & -0.005 & -0.218 & -0.077 & -0.217 & -0.005 & -0.171 & 0.141 \\
\hline State $4-7$ & 0.056 & -0.048 & 0.034 & 0.014 & -0.099 & 0.069 & -0.005 & 0.037 & 0.015 & 0.004 & 0.214 \\
\hline State $5-7$ & 0.101 & 0.009 & 0.157 & -0.163 & -0.084 & -0.118 & -0.0222 & -0.036 & -0.027 & -0.001 & 0.248 \\
\hline$-2 \mathrm{LL}$ & 2869.38 & & & & & & & & & & \\
\hline
\end{tabular}

Table 3. Effects of different variables on transition intensities based on viral load states 
of the next state an individual goes to, after leaving state $i$. If $q_{i i}>0$, then given that there is a jump to a different state, implies the patient will not stay in state $i$, the patient makes a jump out of state $i$ resulting in $R_{i i}=$ 0 , and if $q_{i i}=0$, then the patient will never leave state $i$, implying that $R_{i i}=1$ (in states 6 and 7). The computed matrix of probabilities of each state being next (also known as the jump process), together with the mean sojourn times in each state, fully define a continuous-time Markov model. This is a more intuitively meaningful description of a model than the transition intensity matrix. The matrix for the probabilities that the next state after state $i$ is state $j$ is approximated as $p_{i j}=\frac{q_{i j}}{\lambda_{i} \text { after state } i \text { is, for each } i \text { and }}$ $j$, such that $i \neq j$ is the force of transition from state $i$ to state $j$ and $q_{i i}$ is the total force of transition out of state $i$. For example, the probability that state 2 is next given that the patient is initially in state 1 is given by

$p_{12}=\frac{q_{12}}{\lambda_{1}}=\frac{0.410}{0.410+0.010+0.037} \approx 0.897$ as shown in Table 4 .

The results in Table 4 show an increase in the probabilities of transition to death with increasing VL states, resulting in patients with VL levels $>500000$ copies $/ \mathrm{mL}$ having the highest chances of transitions to death. For patients with a VL $>10000$ copies $/ \mathrm{mL}$ (state 3 , state 4 and state 5), probabilities of viral suppression to levels between 50 and 10000 copies/mL (state 2) are higher compared with transitions to any other states. The results generally show higher chances of transitions to viral suppression than viral rebound, an indication of treatment efficacy.

Fig. 2 shows the plots of percentage prevalence in each state from time of treatment commencement to the end of the study period. The model gives an almost perfect fit of the observed data. The plots show an increase in percentage prevalence during the first 0.5 years for state 1 or the undetectable VL state. After 1.5 years, there is a slight drop in percentage prevalence from state 1 . This could be attributed to factors such as developing active TB on cART, virological failure, peripheral neuropathy and lactic acidosis, as shown in Table 3.

\section{Discussion}

In this study, a continuous-time nonhomogeneous Markov model was used to model the progression of HIV/TB co-infected patients receiving cART at an SA rural clinic. Non-homogeneity of transition intensities

Table 4. Probability of each state being next (jump chain)

\begin{tabular}{llllllll}
\hline & \multicolumn{7}{c}{ To $(j)$} \\
\cline { 2 - 8 } From $(i)$ & State 1 & State 2 & State 3 & State 4 & State 5 & State 6 & State 7 \\
\hline State 1 & 0 & 0.897 & 0 & 0 & 0 & 0.022 & 0.081 \\
State 2 & 0.703 & 0 & 0.257 & 0 & 0 & 0.010 & 0.031 \\
State 3 & 0.225 & 0.645 & 0 & 0.102 & 0 & 0.009 & 0.019 \\
State 4 & 0.086 & 0.787 & 0.023 & 0 & 0.072 & 0.022 & 0.009 \\
State 5 & 0.102 & 0.582 & 0 & 0.266 & 0 & 0.025 & 0.024 \\
State 6 & 0 & 0 & 0 & 0 & 0 & 1 & 0 \\
State 7 & 0 & 0 & 0 & 0 & 0 & 0 & 1
\end{tabular}

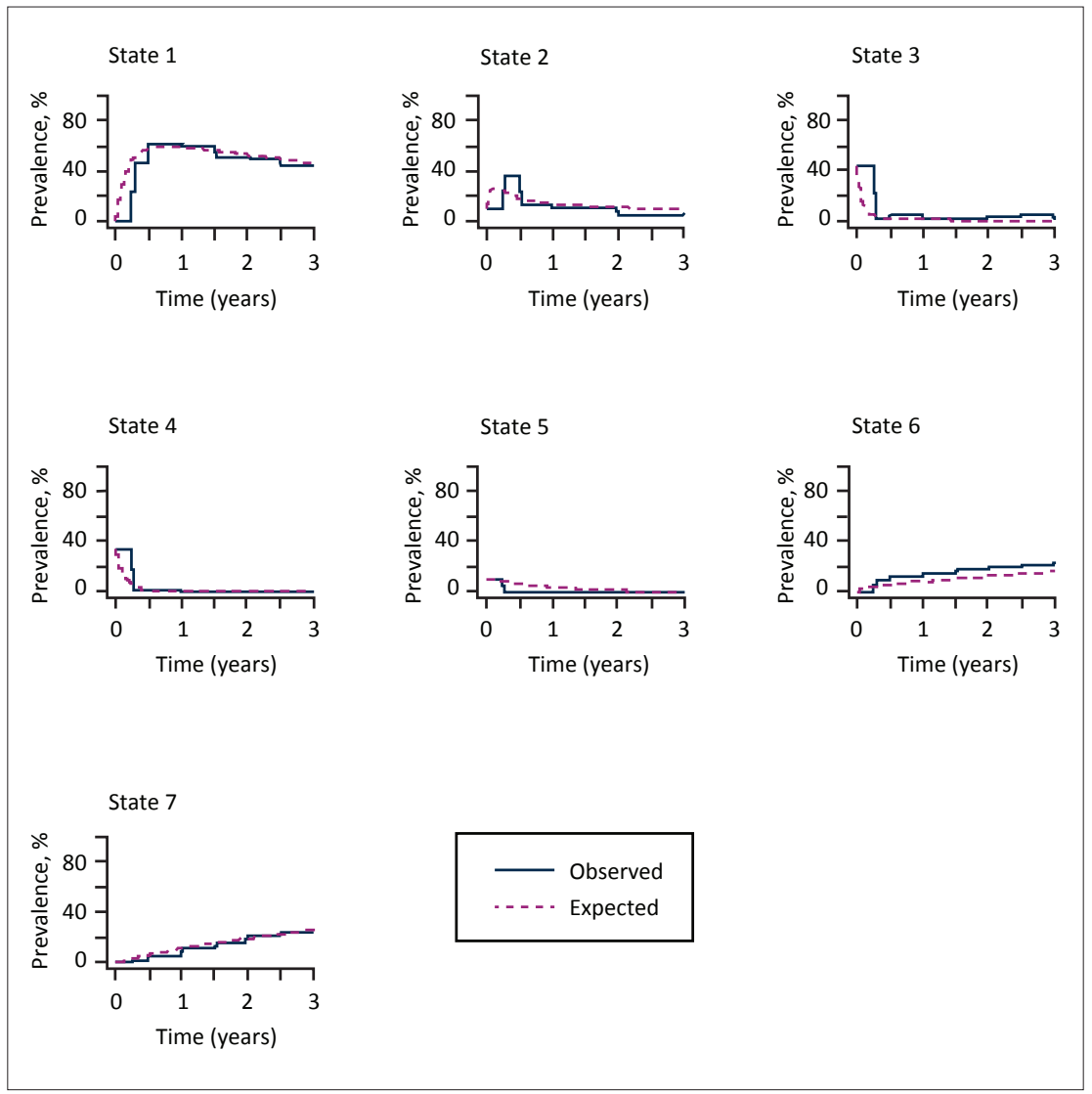

Fig. 2. Percentage prevalence in each state from time of treatment commencement to 3 years after commencement.

was approached using a piecewise constant model, allowing transitions to vary between different time segments. The effects of VL prior to treatment initiation, gender, developing active TB while on CART, having TB at enrolment, peripheral neuropathy, lactic acidosis, virological failure and treatment failure on HIV/AIDS progression defined by transition intensities were assessed.

Results from the analysis show a bidirectional movement between HIV states, thus revealing a possibility of viral rebound and viral suppression. However, transitions to suppressed VLs are higher than transitions to viral rebound. This is quite encouraging, as it shows efficacy of
ART in reducing VL as expected. Results also show that, from any VL state, an undetectable VL state can be attained. The attainment of undetectable VL levels varies from state to state, with patients at lower VL levels having better chances of attaining undetectable VLs than patients at higher VL levels. The undetectable viral load is achieved during the first 0.5 years of treatment uptake, according to our findings, which show a rapid increase in state 1 (undetectable VL) prevalence within the first 0.5 years.

Findings from this study reveal that, although the undetectable VL state can be attained from any HIV state, there are some 
factors that increase the rate of viral rebound from undetectable levels to VLs between 50 and 10000 copies $/ \mathrm{mL}$. The results reveal increased rates (by $>7$-fold) of virological failure from undetectable VL levels to VLs between 50 and 10000 copies/mL. This increase is attributed to the development of active TB while on ART, lactic acidosis and peripheral neuropathy, with the development of active TB on cART the major cause. However, if TB is detected at enrolment, rates of viral rebound from undetectable levels are reduced. This finding is corroborated by studies by $\mathrm{UNAIDS}^{[22]}$ suggesting that early diagnosis and timely treatment of TB reduce the risk of virological rebound.

The risk of virological failure from between 50 and 10000 copies/ $\mathrm{mL}$ to a state between 10000 and 100000 copies $/ \mathrm{mL}$ increased by $\sim 3.5$ times for patients who had TB at enrolment, developed TB on cART, or had lactic acidosis, treatment failure or a VL baseline $>10000$ copies $/ \mathrm{mL}$ at enrolment. Developing active TB on cART had the highest contributory effect to virological failure.

Our findings reveal that patients generally experience virological failure in state 4 (i.e. VL baseline between 100000 and 500000 copies $/ \mathrm{mL}$ ). These patients experience a viral rebound to a VL level $>500000$ copies $/ \mathrm{mL}$ (state 5). Virological failure coupled with development of active TB while on ART increases rates of HIV/AIDS progression as well as mortality from HIV/AIDS after achieving an undetectable VL. Bulage et al. ${ }^{[3]}$ suggested that having active TB increases the odds of virological non-suppression.

The present study also revealed that patients who developed active TB while on cART experienced increased rates of transitions to death irrespective of their HIV/AIDS state. The highest rates were experienced when VL levels were between 50 and 10000 copies $/ \mathrm{mL}$ where patients who developed active TB on cART were 3.2 times more likely to experience death than patients who did not develop active TB. Our findings are in congruence with those of Bekker and Wood $^{[23]}$ and von Reyn et al. ${ }^{[24]}$ which show increased mortality rates in HIV patients with virological failure and co-infected with TB. Bekker and Wood ${ }^{[23]}$ also observed that the onset of TB in HIVinfected patients is associated with an increased risk of AIDS and death. We therefore propose regular monitoring of HIV patients coinfected with TB for virological failure. This should be done every 2 months, and decisions should be made on alternative treatment approaches to prevent potential mortality.

\section{Conclusions}

Our findings reveal the importance of time in monitoring HIV/ AIDS progression for patients with virological failure as well as TB co-infection. The piecewise constant approach to non-homogeneous Markov modelling used shows two different trends in HIV/AIDS progression, i.e. a sharp increase in state 1 (undetectable VL level) prevalence in the first 0.5 years of treatment followed by a slowly decreasing prevalence trend thereafter. Not surprisingly, the model confirms that virological failure increases the risk of death. However, it suggests that while TB at the time of ART initiation does not increase the risk of viral rebound, the development of active TB after initiation of ART does increase this risk. This finding highlights the importance of improving VL monitoring, especially in people at risk of TB, and addressing unsuppressed VLs in this category of patients.

\section{Declaration. None.}

Acknowledgements. We are grateful for the co-operation of the study participants at the time of data collection.
Author contributions. CS drafted the manuscript. POB provided the data used for the analysis. CS, DC and POB contributed to the analysis and interpretation of the data. All authors participated in critical revision of the manuscript drafts and approved the final version.

Funding. PB's research was supported by the South African Medical Research Council through funding received from the South African National Treasury, the South African National Research Foundation (GUN109312, GUN86037) and the University of Venda. The views expressed are solely the responsibility of the authors and do not necessarily represent the official views of the South African Medical Research Council, the National Research Foundation or the University of Venda.

Conflicts of interest. None.

1. Joint United Nations Programme on HIV/AIDS (UNAIDS). UNAIDS data 2018. https://www unaids.org/sites/default/files/media_asset/unaids-data-2018_en.pdf (accessed 3 April 2019).

2. Pawlowski A, Jansson M, Sköld M, Rottenberg ME, Källenius G. Tuberculosis and HIV co-infection. PLoS Pathog 2012;8(2):e1002464. https://doi.org/10.1371/journal.ppat.1002464

3. Bulage L, Ssewanyana I, Nankabirwa V, et al. Factors associated with virological nonsuppression among HIV-positive patients on antiretroviral therapy in Uganda, August 2014 - July 2015. BMC among HIV-positive patients on antiretroviral therapy in Uganda,
Infect Dis 2017;17:326. https://doi.org/10.1186/s12879-017-2428-3

4. Martinson NA, Hoffmann CJ, Chaisson RE. Epidemiology of tuberculosis and HIV: Recent advances in understanding and responses. Proc Am Thorac Soc 2011;8(3):288-293. https://doi.org/10.1513 pats.201010-064WR

5. Bruisker T, Dufour MK, Myers JJ. Recall of nadir CD4 cell count and most recent HIV viral load among HIV-infected, socially marginalized adults. AIDS Behav 2015;19(11):2108-2116. https://doi. org/10.1007/s10461-015-1018-x.

6. Cohen K, Meintjes G. Management of individuals requiring ART and TB treatment. Curr Opin HIV AIDS 2010;5(1):61-69. https://doi.org/10.1097/COH.0b013e3283339309

7. World Health Organization. Global Tuberculosis Control. WHO Report 2001. Geneva: WHO, 2001 https://apps.who.int/iris/bitstream/handle/10665/63835/WHO_CDS_TB_2001.287.pdf (accessed 2 April 2019)

8. Day JH, Grant AD, Fielding KL, et al. Does tuberculosis increase HIV load? J Infect Dis 2004;190:1677-1684. https://doi.org/10.1086/424851

9. Sigaloff KCE, Hamers RL, Wallis CL, et al. Unnecessary antiretroviral treatment switches and accumulation of HIV resistance mutations: Two arguments for viral load monitoring in Africa. J Acquir Immune Defic Syndr 2011;58:23-31. https://doi.org/10.1097/QAI.0b013e318227fc34

10. Lee S, Ko J, Tan X, et al. Markov chain modelling analysis of HIV/AIDS progression: A race based forecast in the United States. Indian J Pharm Sci 2014;76(2):107-115.

11. Chenand B, Zhou XH. Non-homogeneous Markov process models with informative observations with an application to Alzheimer's disease. Biom J 2011;53(3):444-463. https://doi.org/10.1002/ bimj. 201000122

12. Kalbfleisch JD, Lawless JF. The analysis of panel data under a Markov assumption. J Am Stat Assoc 1985;80(392):863-871. https://doi.org/10.1080/01621459.1985.10478195

13. Duffy MJ, Blaser J, Duggan C, et al. Assay of matrix metalloproteases types 8 and 9 by ELISA in human breast cancer. Br J Cancer 1995;71:1025-1028, PMID:7734294. https://doi.org/10.1038/ bjc.1995.197

14. Yen AMF, Chen THH. Mixture multi-state Markov regression model. J Appl Stat 2007;34(1):11-21. https://doi.org/10.1080/02664760600994711

15. Pan W, Kastin AJ, Tumor necrosis factor and stroke: Role of the blood-brain barrier. Prog Neurobiol 2007;83(6):363-374. https://doi.org/10.1016/j.pneurobio.2007.07.008

16. Marshall G, Jones RH. Multi-state Markov models and diabetic retinopathy. Stat Med 1995;14(18):1975-1983. https://doi.org/10.1002/sim.4780141804

17. Lindsay JC, Ryan LM. A three-state multiplicative model for rodent tumorigenicity experiments. Appl Stat 1993;42(2):283-300. https://doi.org/10.2307/2986233

18. Stead D. Southern African HIV Clinicians Society Guidelines 2017. https://sahivsoc.org/Files HIV\%20clinicians\%20ADULT\%20GUIDELINES\%202017\%20-\%20David\%20Stead.pdf (accessed 18 June 2019).

19. Gunda DW, Kidenya BR, Mshana SE, Kilonzo SB, Mpondo BCT. Accuracy of WHO immunological criteria in identifying virological failure among HIV-infected adults on first line antiretrovira therapy in Mwanza, north-western Tanzania. BMC Res Notes 2017;10:45. https://doi.org/10.1186/ s13104-016-2334-6

20. Dalakas MC. Peripheral neuropathy and antiretroviral drugs. J Peripher Nerv Syst 2001;6(1):14-20. https://doi.org/10.1046/j.15298027.2001.006001014.x

21. Saint-Pierre P, Combescure C, Daurès JP, et al. The analysis of asthma control under a Markov Saint-Pierre P, Combescure C, Daurès JP, et al. The analysis of as
assumption with use of covariates. Stat Med 2003;22(24):3755-3770.

22. Joint United Nations Programme on HIV/AIDS (UNAIDS). UNAIDS data 2017. https://www. unaids.org/sites/default/files/media_asset/20170720_Data_book_2017_en.pdf (accessed 10 May 2019).

23. Bekker LG, Wood R. TB and HIV co-infection: When to start antiretroviral therapy: Guidelines on when to start therapy in TB and HIV co-infection. CME 2011;29(10). http://www.cmej.org.za/index. php/cmej/article/view/2323/2035 (accessed 5 April 2019).

24. Von Reyn CF, Mtei L, Arbeit RD, et al. Prevention of tuberculosis in Bacille Calmette-Guérinprimed, HIV-infected adults boosted with an inactivated whole-cell mycobacterial vaccine. AIDS 2010; 24(5):675-685. https://doi.org/10.1097/QAD.0b013e3283350f1b 